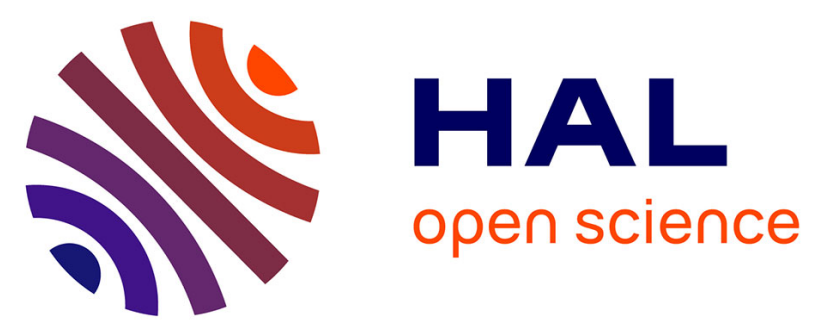

\title{
Damage-to-fracture transition through an Eikonal Non-Local (ENL) continuum damage formulation with embedded strong discontinuities
}

Flavien Thierry, Giuseppe Rastiello, Cédric Giry, Fabrice Gatuingt

\section{- To cite this version:}

Flavien Thierry, Giuseppe Rastiello, Cédric Giry, Fabrice Gatuingt. Damage-to-fracture transition through an Eikonal Non-Local (ENL) continuum damage formulation with embedded strong discontinuities. 10th International Conference on Fracture Mechanics of Concrete and Concrete Structures, FraMCoS-X, Jun 2019, Bayonne, France. 10.21012/FC10.233386 . hal-02436622

\section{HAL Id: hal-02436622 \\ https://hal.science/hal-02436622}

Submitted on 13 Jan 2020

HAL is a multi-disciplinary open access archive for the deposit and dissemination of scientific research documents, whether they are published or not. The documents may come from teaching and research institutions in France or abroad, or from public or private research centers.
L'archive ouverte pluridisciplinaire $\mathbf{H A L}$, est destinée au dépôt et à la diffusion de documents scientifiques de niveau recherche, publiés ou non, émanant des établissements d'enseignement et de recherche français ou étrangers, des laboratoires publics ou privés. 


\title{
DAMAGE-TO-FRACTURE TRANSITION THROUGH AN EIKONAL NON-LOCAL (ENL) CONTINUUM DAMAGE FORMULATION WITH EMBEDDED STRONG DISCONTINUITIES
}

\section{FLAVIEN THIERRY ${ }^{\dagger}$, GIUSEPPE RASTIELLO ${ }^{\ddagger}$, CEDRIC GIRY $^{\dagger}$ AND FABRICE GATUINGT $^{\dagger}$}

\author{
${ }^{\dagger}$ LMT, ENS Paris-Saclay, CNRS, Université Paris-Saclay \\ 94235 Cachan, France \\ e-mail: \{flavien.thierry;cedric.giry;fabrice.gatuingt\}@ens-paris-saclay.fr
}

‡DEN, Service d'études mécaniques et thermiques (SEMT), CEA, Université Paris-Saclay

91191 Gif-sur-Yvette, France

e-mail: giuseppe.rastiello@cea.fr

Key words: Eikonal Non Local damage, Regularization, Evolving non local interactions, Strain localization, Embedded discontinuities

\begin{abstract}
The Eikonal Non-Local damage model (ENL) is an integral-based regularization method with damage-dependent non-local interactions. This contribution introduces the transition from an ENL formulation to a strong discontinuity model in a one-dimensional case. By introducing the formation of a cohesive crack in highly damaged areas, this new formulation seeks to solve problems of objectivity of results with respect to the mesh size for regularization methods with evolution of non-local interactions leading to strain localization.
\end{abstract}

\section{INTRODUCTION}

Predicting the cracking of quasi-brittle materials is a major challenge in the study of the robustness and durability of civil engineering structures. Damage models are part of the classic methods for dealing with these problems. The implementation of this type of models in the finite element method results in a pathological dependence on the mesh size. The introduction of a characteristic length in the formulation makes it possible to find objectivity of the results with regard to the spatial discretization.

Among the classical regularization methods, the Integral Non-Local (INL), developed by Pijaudier-Cabot and Bazant [1], proposes to control the damage by a non-local variable calculated as a spatial average of its local values. However, the nature of the INL method (based on Euclidean distances) can lead to non-physical interactions, for example through cracks or strongly damaged zones. To address this problem, several methods seek to adjust the non-local interactions using the mechanical state of the material. For example, Giry et al. [2] and Pijaudier-Cabot et al. [3] propose to weight non-local interactions according to the stress or damage level, respectively.

The non-local eikonal method (ENL) [4] proposes to weight non-local interactions by the lengths of the geodesics in a Riemannian space curved by damage. In this case, the field of geodesic distances is a solution of an eikonal equation in which the metric is damage dependent. Rastiello et al. [5] have recently shown that this formulation allows avoiding diffusion of the damage zone observed for the INL for- 
mulation. Regularization properties are preserved up to a high level of damage. However, for all these advanced methods, mesh dependence can be observed in the case of the formation of very severely damaged areas. Indeed, non-local interactions tend to decrease with the evolution of damage, and the material response becomes local in these areas.

This contribution proposes to improve the regularization properties of the ENL method by considering severely damaged areas as cohesive cracks. Thus the ENL method is coupled to a model with strong discontinuity. The transition from the non-local method to the high discontinuity model is first presented, then the regularization properties of this formulation are illustrated using a one-dimensional numerical study on a bar loaded in tension.

\section{EIKONAL NON LOCAL FORMULA- TION (ENL)}

In an isotropic one-dimensional elastic body $\Omega$, it is possible to quantify the degradation of the material for any point $x \in \Omega$ using a scalar damage variable $D(x) \in[0,1][6]$. Beyond the elastic limit of the material, degradation begins, and the damage variable increases irreversibly. For a local damage model, the evolution of the damage variable $D$ is usually controlled by a variable $\varepsilon_{e q}(x)$ expressed as a function of quantities evaluated at the point $x$.

\subsection{Integral Non-Local Method (INL)}

For a non-local model, damage evolves according to a non-local variable $\varepsilon_{e q}^{n l}(x)$ :

$$
D(x)=g\left(\varepsilon_{e q}^{n l}(x)\right)
$$

In particular, in the INL method [1], the nonlocal variable corresponds to a spatial average of its local values:

$$
\begin{aligned}
\varepsilon_{e q}^{n l}(x) & =\frac{1}{V(x)} \int_{\Omega} \phi\left(\frac{\ell_{x y}}{\ell_{c}}\right) \varepsilon_{e q}(y) d y \\
V(x) & =\int_{\Omega} \phi\left(\frac{\ell_{x y}}{\ell_{c}}\right) d y
\end{aligned}
$$

where $\phi$ is a decreasing weighting function of the distance $\ell_{x y}$ separating the two points $x$ and $y$. This function is generally chosen as a Gaus$\operatorname{sian} \phi\left(\xi=\ell_{x y} / \ell_{c}\right)=\exp \left(-4 \xi^{2}\right)$. Parameter $\ell_{c}$ corresponds to a characteristic distance defining the size of the domain outside which non-local interactions are negligible.

\subsection{Non-local method based on internal time}

For the INL method, the distance is simply the Euclidean distance between the two points. This distance remains fixed over time. This implies that two points can continue to interact from a non-local point of view after a crack appears between them. The principle of the ENL method is to take into account the damage field when calculating distances and thus avoid nonphysical interaction problems.

By writing the non-local problem in time rather than distance, Desmorat and Gatuingt [7] propose to weight the non-local interactions according to the propagation time $\tau_{x y}$ of an elastic wave in the damaged environment:

$$
\varepsilon_{e q}^{n l}(x)=\frac{1}{V(x)} \int_{\Omega} \phi\left(\frac{\tau_{x y}}{\tau_{c}}\right) \varepsilon_{e q}(y) d y
$$

where $\tau_{c}$ is a characteristic time. It represents the time required for an elastic wave to cover the distance $\ell_{c}$ in the undamaged environment. With $c_{0}$ the initial velocity of the wave, the characteristic time is related to the characteristic length of the INL method: $\tau_{c}=\ell_{c} / c_{0}$.

In an elastic medium, the propagation time of a wave is related to the velocity of the wave and this velocity is proportional to the square root of the stiffness of the material. In the case of a damaged elastic medium, the stiffness of the material is reduced by damage. As a result, the propagation time of a wave between two points separated by a damaged area will tend to increase, and non-local interactions between these points will be reduced.

This time-based formulation naturally introduces the effect of damage on the non-local behavior of the material. However, from a numerical point of view, it is not reasonable (in two- or three-dimensional settings) to perform a 
dynamic wave propagation calculation for each loading step and each point in the domain.

\subsection{Eikonal Non-local method (ENL)}

Using a high-frequency approximation of the wave equation, Desmorat et al. [4] show that the non-local propagation time problem can be written as a function of the effective distances $\tilde{\ell}_{x y}$ corresponding to the geodesic lengths in a Riemannian space curved by damage. In a unidimensional setting, the effective distance field is then the solution of the following eikonal equation:

$$
\begin{aligned}
\sqrt{1-D(y)}\left|\frac{\mathrm{d}}{\mathrm{d} y} \tilde{\ell}_{x y}(y)\right| & =1 \\
\tilde{\ell}_{x y}(y=x) & =0
\end{aligned}
$$

In this case, non local damage follows equation (4). For each time-step, the effective distance is calculated according to the eikonal equation.

\subsection{Regularization features of the ENL method}

A bar $\Omega$ of length $L$ and cross section $A$ (figure 11) loaded in tension is studied using numerical finite element simulations. The bar material is modeled with an ENL damage model. In order to trigger the localization, a stiffness defect is introduced at the middle of the bar through a weakened finite element. To describe the system's snap-back response in the load post-peak phase, the applied displacement at the end of the bar is indirectly controlled by imposing a constant rate of variation to the deformation of the weakened finite element [8].

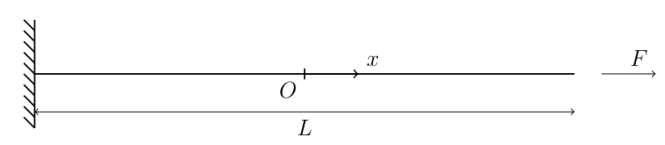

Figure 1: Effective distance field in a notched plane

The material is initially linear elastic. When the yield strength is reached, degradation begins to develop. In the calculation, a decreasing exponential evolution law is used to quantify the development of degradation:

$$
D=g(\kappa)=1-\frac{\varepsilon_{0}}{\kappa} \exp \left(-\frac{\left\langle\kappa-\varepsilon_{0}\right\rangle_{+}}{\varepsilon_{c}-\varepsilon_{0}}\right)
$$

with:

$$
\kappa=\max \left(\kappa, \varepsilon_{e q}^{n l}\right)
$$

In equation (7), $\varepsilon_{0}$ represents the threshold deformation corresponding to the activation of the damage process and $\varepsilon_{c}$ is a critical deformation level. This parameter controls the energy dissipated in the post-peak stress phase. The non-local interaction distances between the integration points of the finite element mesh are updated at the beginning of each loading step. In the one-dimensional context considered here, the effective distance $\tilde{\ell}_{x y}$ between each pair $(x, y)$ of Gauss points is computed as:

$$
\tilde{\ell}_{x y}=\int_{\min (x, y)}^{\max (x, y)} \frac{1}{\sqrt{1-D(z)}} d z
$$

Material parameters used in computations are given in Table 1

Table 1: Geometrical and material parameters

\begin{tabular}{ccc}
\hline$L$ & 100 & $\mathrm{~mm}$ \\
\hline$A$ & $5 \times 10^{-3}$ & $\mathrm{~m}^{2}$ \\
\hline$E$ & 100 & $\mathrm{MPa}$ \\
\hline$\varepsilon_{0}$ & $1 \times 10^{-4}$ & - \\
\hline$\varepsilon_{c}$ & $10 \times \varepsilon_{0}$ & - \\
\hline$\ell_{c}$ & 20 & $\mathrm{~mm}$ \\
\hline
\end{tabular}

The results obtained using the ENL formulation are plotted in figures 2 and 3 for three different mesh sizes (41, 81 and 161 bar-type linear finite elements). The force-displacement response curves (figure 2) show that, for low damage levels, the ENL method provides the same structural behavior as the classical INL method because the geodesic distances are close to Euclidean distances. The damage profiles are drawn on figure 3 for several loading stations.

When damage reaches values close to unity, the geodesic distances are much higher than the 
Euclidean distances and the non-local interaction area becomes very small. Once this condition is achieved, the global response corresponds to the one obtained using a local damage model, and a residual dependence on the mesh size is observed. The same kind of response was found by [5]. In other words, the residual energy still available in the weakened finite element during the localization phase can only be dissipated in a local manner.

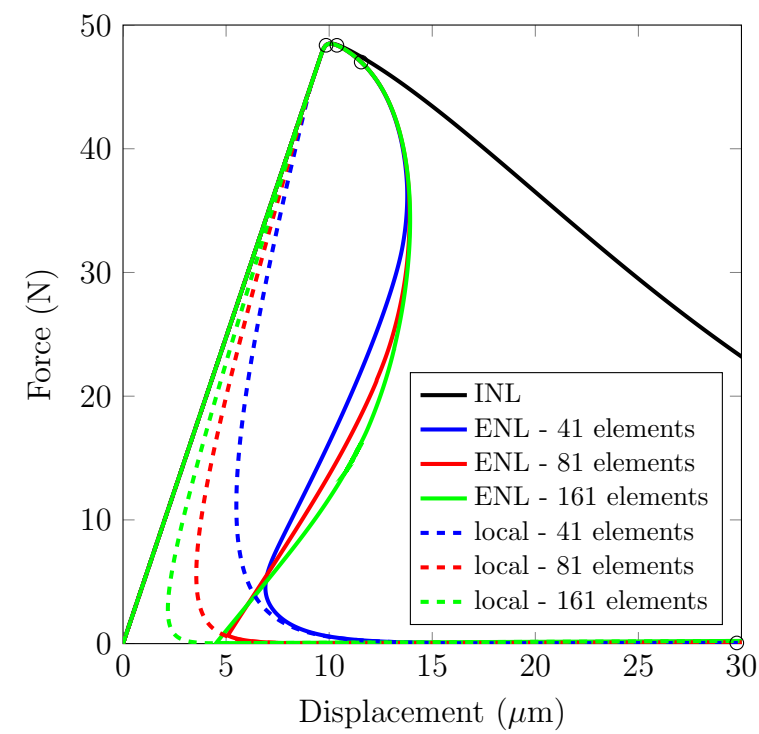

Figure 2: Regularization properties of the ENL method: force-displacement responses computed for three different mesh sizes

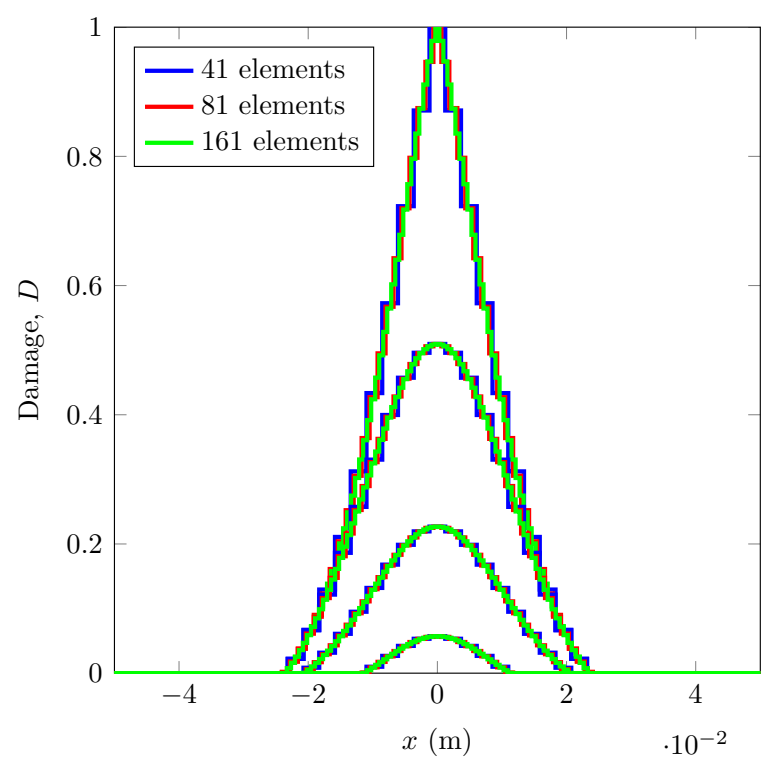

Figure 3: Damage profiles in the bar using the ENL method (three different mesh sizes).

\section{ENL FORMULATION WITH EMBED- DED STRONG DISCONTINUITIES}

This section proposes a first attempt in modeling the failure of quasi-brittle materials using an ENL damage law coupled with a strongdiscontinuity formulation [9.-11]. In this way, non-local damage allows for modeling diffuse degradation leading to the localization into a cohesive crack. This approach is consistent with the physics of the problem. Moreover, from a numerical point of view, the formulation of this problem in the framework of the embedded finite element method makes it possible to eliminate the residual mesh dependence previously observed.

\subsection{ENL damage to strong discontinuity transition model}

During the first stages of the damaging process, the behavior of the material follows equations (4) and (5). The damage-to-fracture transition time (at $x_{0} \in \Omega$ ) is arbitrarily chosen as the time when $D\left(x_{0}\right)$ reaches a critical damage value $D_{c r}$. At the discontinuity level, a tractionseparation law (10) gives the relationship between the displacement jump $\left(u^{d}\right)$ and the co- 
hesion stress $(t)$ :

$$
t=g_{d}\left(u^{d}\right) \quad x=x_{0}
$$

Finally, the following traction continuity continuity established the equilibrium across the discontinuity:

$$
\sigma-t=0 \quad x=x_{0}
$$

Once this condition is attained, material points close to the crack still behave according to the ENL formulation. Their interaction with $x_{0}$ are however reduced by considering that $D\left(x_{0}\right)=1$ in (9).

The numerical formulation of the previous problem is achieved in the context of the Embedded Finite Element Method [12]. The additional degrees of freedom related to the strong discontinuity (i.e., displacement jumps) are interpolated using the Heaviside function and the standard shape functions of the finite element. Then, these degrees of freedom are statically condensed at the finite elements level, which makes it possible to solve the problem in a staggered way. The global degrees of freedom (i.e., nodal displacements) are computed by solving the global equilibrium equation by freezing elemental crack openings. These elemental degrees of freedom are then updated element-byelement according to the global displacement field that has just been calculated.

\subsection{Regularization features of the ENL for- mulation with embedded strong discon- tinuities}

Consider the previous example of a onedimensional bar. The material has an initially ENL behavior. The transition to a high discontinuity model occurs when $D>D_{c r}$ in the weakened finite element. For this element the traction-separation law is defined as:

$$
t=g_{d}\left(u^{d}\right)=\exp \left(-\frac{\sigma_{c r}}{G_{f}} u^{d}\right)
$$

where $G_{f}$ is the dissipated energy in the cohesive law, $\sigma_{c r}=\left(1-D_{c r}\right) E \varepsilon_{c r}$ denotes the stress in the bar at the time of transition and $\varepsilon_{c r}$ the corresponding strain in the weakened element. Geometrical and material parameters used in computations are the same as in examples illustrated in the previous section. The additional parameter $D_{c r}$ is chosen equal to 0.8 .

Figure 4 gives the results obtained for different mesh sizes and three values of the crack energy parameter $G_{f}$. These results validate the regularization properties of the ENL method with the transition to strong discontinuity, from damage initiation to complete structural degradation.

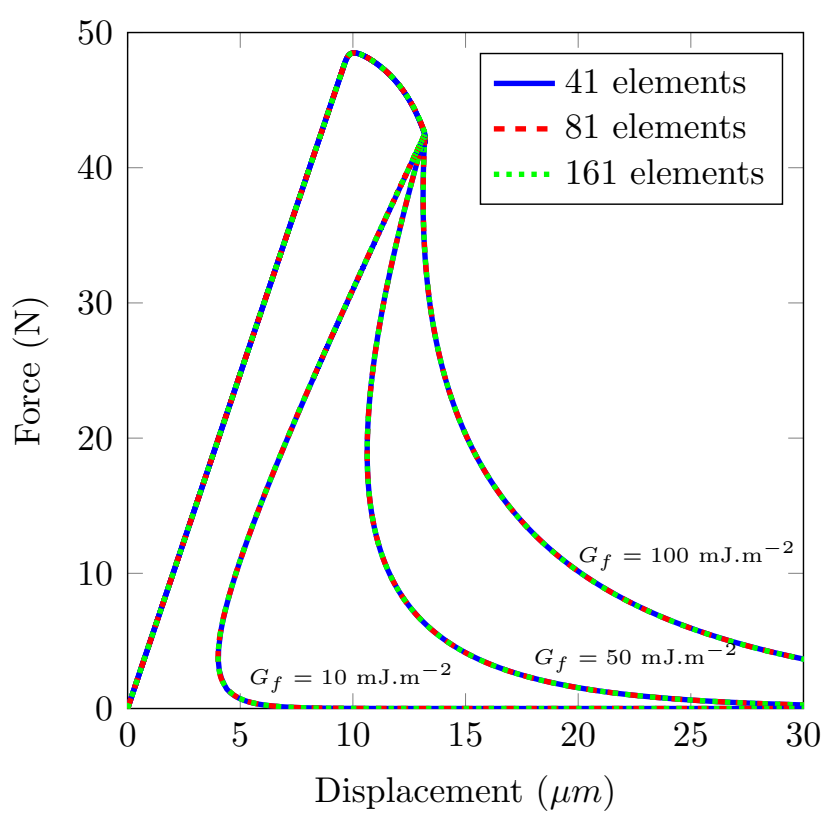

Figure 4: Regularization properties of the ENL damage-to-fracture transition method: forcedisplacement responses computed for three different mesh sizes

Figure 5 shows the evolution of the strain in the bulk material $\left(\varepsilon_{b}=\varepsilon-u^{d} / L_{e}\right.$, where $L_{e}$ refers to the length of the finite element) and the apparent deformation due to the crack opening $\left(u^{d} / L_{e}\right)$ in the weakened element of a mesh comprising 41 finite elements. The strain evolution in a finite element directly next to the localized one is also considered. For the same elements, damage evolutions are provided in Figure 6. As expected, after the introduction of the discontinuity, $\varepsilon_{b}$ decreases whereas $u^{d} / L_{e}$ progressively increases and tends to $\varepsilon$. During 
this phase, the damage field along the bar does not evolve. Notice, however, that the damage field is not artificially frozen after the crack is activated. In other words, such response results from the progressive unloading of the bulk only.

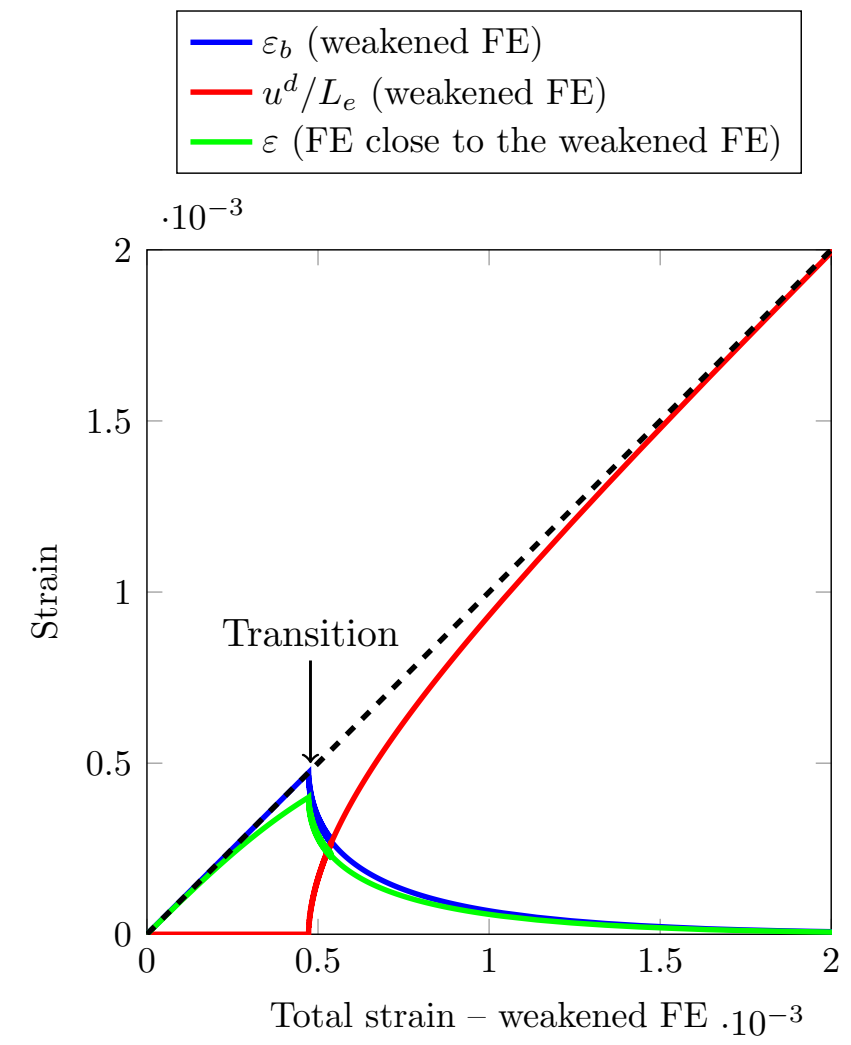

Figure 5: Strain evolution in the weakened element (mesh comprising 41 elements).

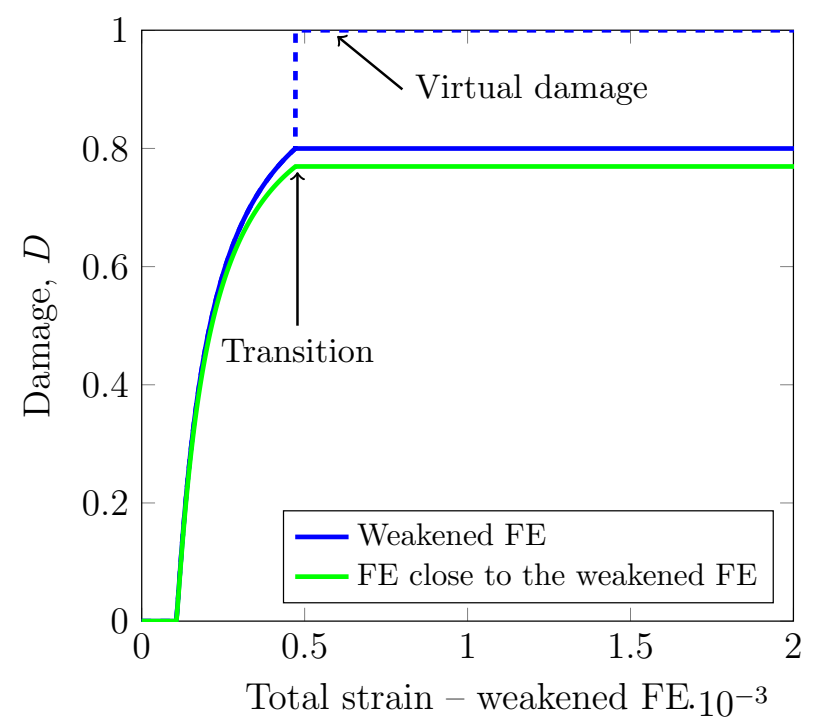

Figure 6: Damage evolution for the ENL formulation with embedded strong discontinuities (mesh comprising 41 elements).

\section{CONCLUSIONS}

A first attempt in modeling the transition from an ENL damage model to a strong discontinuity formulation was presented. First results in $1 \mathrm{D}$ show that the introduction of discontinuity in highly damaged areas makes it possible to restore the objectivity of the results when the localization area is reduced to the size of a single finite element. In view of this work, the definition of an objective quantification of the energy dissipated during the cracking process until failure is under development. Furthermore, objective damage-to-fracture transition criteria are under investigation.

\section{ACKNOWLEDGMENTS}

This work was partly supported by the SEISM Institute (http://www. institut-seism.fr).

\section{REFERENCES}

[1] Gilles Pijaudier-Cabot and Z. P. Bazant. Non local Damage Theory. Journal of engineering mechanics, 113(10):15121533, 1987.

[2] Cédric Giry, Frédéric Dufour, and Jacky Mazars. Stress-based nonlocal dam- 
age model. International Journal of Solids and Structures, 48(25-26):34313443, 2011.

[3] Gilles Pijaudier-Cabot, Khalil Haidar, and Jean François Dubé. Non-local damage model with evolving internal length. International Journal for Numerical and Analytical Methods in Geomechanics, 28(78):633-652, 2004.

[4] R. Desmorat, F. Gatuingt, and M. Jirásek. Nonlocal models with damage-dependent interactions motivated by internal time. Engineering Fracture Mechanics, 142:255-275, 2015.

[5] Giuseppe Rastiello, Cédric Giry, Fabrice Gatuingt, and Rodrigue Desmorat. From diffuse damage to strain localization from an Eikonal Non-Local (ENL) Continuum Damage model with evolving internal length. Computer Methods in Applied Mechanics and Engineering, 331:650-674, 2018.

[6] J Lemaitre and Rodrigue Desmorat. Engineering Damage Mechanics. Ductile, Creep, Fatigue and Brittle Failure. 01 2005.

[7] Rodrigue Desmorat and Fabrice Gatuingt. Introduction of an internal time in nonlocal integral theories. Computational Modelling of Concrete Structures, pages 121128, 2007.

[8] Giuseppe Rastiello, Francesco Riccardi, and Benjamin Richard. Discontinuity- scale path-following methods for the embedded discontinuity finite element modeling of failure in solids. Computer Methods in Applied Mechanics and Engineering, 349:431-457, 2019.

[9] Fabien Cazes, Michel Coret, Alain Combescure, and Anthony Gravouil. A thermodynamic method for the construction of a cohesive law from a nonlocal damage model. International Journal of Solids and Structures, 46(6):1476-1490, 2009.

[10] Claudia Comi, Stefano Mariani, and Umberto Perego. An extended fe strategy for transition from continuum damage to mode i cohesive crack propagation. International Journal for Numerical and Analytical Methods in Geomechanics, 31:213 - 238, 022007.

[11] Sam Cuvilliez, Frédéric Feyel, Eric Lorentz, and Sylvie Michel-Ponnelle. A finite element approach coupling a continuous gradient damage model and a cohesive zone model within the framework of quasi-brittle failure. Computer Methods in Applied Mechanics and Engineering, 237240:244-259, 2012.

[12] Javier Oliver. Modelling strong discontinuities in solid mechanics via strain softening constitutive equations. i: Fundamentals. ii: Numerical simulation. International Journal for Numerical Methods in Engineering, 39:3575 - 3600, 111996. 